\title{
Review of Nuclear Heating Measurement by Calorimetry in France and USA
}

\author{
C. Reynard-Carette, G. Kohse, J. Brun, M. Carette, A. Volte, A. Lyoussi
}

\begin{abstract}
This paper gives a short review of sensors dedicated to measuring nuclear heating rate inside fission reactors in France and USA and especially inside Material Testing Reactors. These sensors correspond to heat flow calorimeters composed of a single calorimetric cell or of two calorimetric cells at least with a reference cell to obtain a differential calorimeter. The aim of this paper is to present the common running principle of these sensors and their own special characteristics through their design, calibration methods, and in-pile measurement techniques, and to describe multi-sensor probes including calorimeters.
\end{abstract}

Index Terms - Calorimetry, Nuclear Heating, Research Reactors, Fission

\section{INTRODUCTION}

$\mathrm{T}$ HE nuclear energy deposition rate in materials, often called nuclear heating rate or nuclear absorbed dose rate, corresponds to a relevant thermal parameter for fission and fusion reactors. It corresponds to the rate of energy deposited by various interactions between nuclear rays and matter in a mixed $(n, \gamma)$ field. This parameter is required to choose and design accurately thermal and mechanical systems, fluid loops (with or without phase change), instrumentation and probes, but also to interpret in-pile experiments after irradiation and to control ageing of structural materials. Appropriate measurement techniques for this parameter depend on the level and range expected in reactor.

For Zero Power Reactors (ZPRs), nuclear heating quantification can be performed, for instance, by means of OSLDs (Optically Stimulated Luminescent Detectors) and TLDs (Thermo-Luminescent Detectors) [1]. Quantification with these two kinds of sensor is based on three successive steps: out-of-pile calibration, irradiation, and finally postirradiation measurements. Consequently, these sensor types lead to an integrated result for the irradiation duration (nuclear adsorbed dose).

For Material Testing Reactors (MTRs) or other reactors such as Boiling Water Reactors (BWRs), nuclear heating rate quantification is possible thanks to other techniques, more suited to the higher level of nuclear heating occurring inside their experimental channels, and called in-pile calorimeters [223]. This paper focuses mainly on a review of in-pile calorimeters used in French and American reactors. First of all, the operating principle of the calorimetry technique is given. Then characteristics of each sensor are detailed (design, associated instrumentation, calibration, operating protocols and measurement methods). Then some examples of probes including calorimeters are presented.

\section{IN-PILE CALORIMETRY PRINCIPLE}

Calorimetry techniques are used to measure energy/heat in several fields such as material sciences, chemistry, thermodynamics but in nuclear fission field for MTRs a specific running principle is applied due to the high energy deposition rate occurring (up to $20 \mathrm{~kW} / \mathrm{kg}$ in the case of the future MTR under construction in France: Jules Horowitz Reactor). The MTR calorimeters corresponds to non-adiabatic calorimeters (called heat flow calorimeters) which exchange energy continuously with their external surroundings (fluid flow in experimental channels, loops) instead of conventional adiabatic calorimeters.

One major advantage of this calorimeter principle is that it can provide online data while ensuring in-pile calorimeter structure and running integrity.

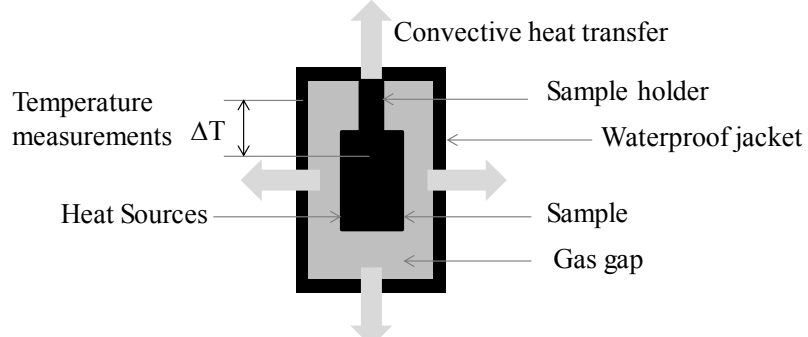

Fig. 1. General scheme of an in-pile calorimeter

A general scheme of an in-pile calorimeter is presented in Figure 1. Quantification of nuclear heating rate in a material sample by means of such sensors is based on measurements of steady temperatures or temperature differences in specific locations inside the sensor body. The specific locations are chosen in order to obtain suitable response and sensitivity, which are controlled by thermal resistances depending on the sensor design, its geometry, its materials and its gas gaps.

The sensor response can be estimated numerically or analytically by solving the heat equation by considering source terms equal to nuclear heating rates and specific boundary conditions corresponding to parietal heat exchanges (empirical correlations to impose a global heat exchange coefficient, for instance). The sensor response can be defined experimentally thanks to out-of-pile or in-pile calibrations. Two methods of calibration can be used, depending on the sensor type.

(kohse@mit.edu). A. Lyoussi is with CEA, DEN, DER, Instrumentation Sensors and Dosimetry Laboratory, Cadarache, F-13108, Saint Paul-lezDurance, France (e-mail: abdallah..lyoussi@cea.fr,)
C. Reynard-Carette, J. Brun, M. Carette and A. Volte are with Aix Marseille Univ, Université de Toulon, CNRS, IM2NP, Marseille, France (e-mail: adrien.volte@im2np.fr, christelle.carette@univ-amu.fr, julie.brun@univamu.fr, michel.carette@univ-amu.fr). G. Kohse is with Nuclear Reactor Laboratory, Massachusetts Institute of Technology, Cambridge, USA 


\section{CALORIMETER TYPES}

Different types of calorimeter are used inside fission reactors. Non-adiabatic calorimeter types can be defined by their assembly. The first category consists of single-cell calorimeters and can be found in Europe in MTRs [2-9] and in USA in BWRs [10-15]. The second class is differential calorimeters and corresponds to French sensors used in MTRs [2, 5, 16-23]. The European calorimeters are used to determine axial distributions of the nuclear heating rate in experimental channels whereas USA calorimeters are used to calibrate local power range detectors dedicated to monitoring the thermal neutron flux during BWR operation.

Schemes of several calorimeters are given in Figure 2.

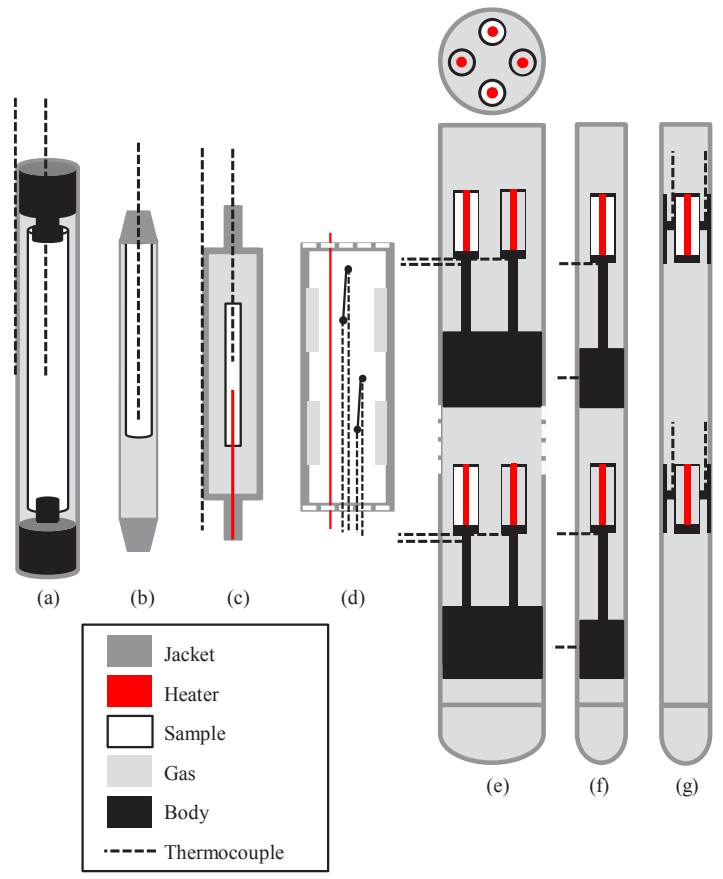

Fig. 2. Schemes of single-cell calorimeters (from (a) to (d)) and differential calorimeters (from (e) to $(\mathrm{g})$ ).

The first category (from (a) to (d)) contains a single calorimetric cell which is in general composed of a sample (white zone), a gas gap (grey zone), a jacket (dark grey zone), a sample holder (black zone), thermocouples (black dotted line) and sometimes a heater (red zone). The nature of the gas and the sample depends on the calorimeter. The Polish calorimeter called KAROLINA (a) has a graphite sample and is filled with helium [2-4, 21]. The gamma thermometer (b) [3-6] contains a stainless steel sample and can be filled with helium or argon according to the targeted nuclear heating level. The number of temperature sensors depends on the calorimeter too. For the gamma thermometer (b) and the USA calorimeter [10-13] (d), only a temperature difference is measured between a hot junction and a cold junction, whereas for the other single-cell calorimeters two absolute temperatures are measured by means of two thermocouples located inside the sample and on the jacket surface, respectively, and then a temperature difference is deduced. The drawing (d) represents two superposed USA calorimeters. Several USA gamma thermometers are superposed to realize fixed in-core measurements at several heights (up to 9 [10]).
The second category (from (e) to (g)) has several calorimetric cells including two kinds of cell [16-23]. This category corresponds to the only current French technique. In the past, single-cell calorimeters having the design (c) were used [7].

The first kind of calorimetric cell called sample cell or measurement cell is similar to a single cell. It contains a material sample. The second cell is used as a reference cell. Its design is the same as the sample cell but it does not contain a material sample. It is used to compensate for the nuclear heating deposition on the calorimetric cell parts distinct from the sample. Consequently, in that case the sensor is called a differential calorimeter because the nuclear heating rate inside the material sample is determined by a heat balance between the sample cell and the reference cell (heat difference thanks to a difference of temperature differences). Three differential calorimeters are presented in Figure 2. The material sample of all these sensors is made of graphite and their gas gap is filled with nitrogen. The specific characteristics of these sensors are induced by the cell number, the sensor-body material and the cell geometry.

The sensor (e) is a five-stage calorimeter [19]. Each stage corresponds to four calorimetric cells (two sample cells + two reference cells). Each cell head is instrumented by a heater and a thermocouple and is linked to a base by a cylindrical rod to produce mainly axial heat evacuation to the external surroundings. This fixed calorimeter leads to the measurement of an axial profile of the nuclear heating rate in five positions without moving the sensor and by taking account of radial flux gradients.

The sensor (f) has two superposed calorimetric cells separated by polished screens (a sample cell in the upper part and a reference cell in the lower part) $[2,5,16-19,22]$. The cell geometry is quite similar to those of the five stage-calorimeter (head + rod + base). This calorimeter type was integrated inside different multi-sensor probes (cf. Section VI). The external diameter of this sensor is smaller than the previous one $(17 \mathrm{~mm}$ against $32 \mathrm{~mm}$ ) but due to the superposition of the two cells the sensor has to be moved to quantify the nuclear heating rate at a given axial position inside the experimental channel in order to avoid the influence of the axial flux gradients. For this calorimeter each cell contains a heater and two thermocouples (located in the upper part of the rod and on the external surface of the base respectively). The nature of the material for its cell structure is aluminum as for that of each five-stage calorimeter cell. However, the type of aluminum depends on the targeted nuclear heating rate (higher thermal conductivity for in-core measurements).

The last differential calorimeter called CALORRE (g) [22, 23], the newest sensor, has two superposed calorimetric cells with a new geometry. The vertical rod is replaced by a horizontal fin linked to a vertical annular fin in contact with the internal surface of the jacket. This geometry leads to a more compact calorimetric cell with radial heat transfer to evacuate the energy instead of axial heat flow. The length of CALORRE cell is reduced to the length of the head of CARMEN cell $(23.1 \mathrm{~mm}$ against $79 \mathrm{~mm}$ ).

Each cell contains a heater and two thermocouples located on the horizontal fin (one close to the head surface and one close to the vertical fin surface). The material structure of each cell is stainless steel instead of aluminum in order to increase the 
thermal resistance and the cell sensitivity due to limitations of the dimensions of the horizontal fin.

\section{CAlorimeter CALIBRATION}

The calibration is a crucial step which depends on the calorimeter type. In the case of a calorimeter with no heater such as KAROLINA calorimeter and gamma thermometers, the calibration method is based on a transient thermal operating protocol in three phases. During the first phase, the calorimeter is put inside a furnace to reach a steady hot temperature (cf. Fig. 3 ). Then during the second phase, the sensor is removed from the furnace to be inserted inside another set-up providing a colder surrounding (such as a fluid flow loop with a lower temperature) [2-4].

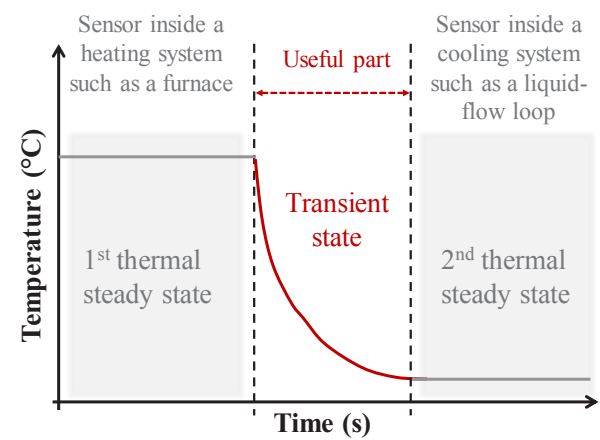

Fig. 3. Scheme of the calibration operating protocol of the calorimeters without heater.

During the last phase, the sensor remains inside the loop to reach a steady cold temperature. Thanks to this calibration operating protocol, the cooling time constant of the sensor can be determined and used to deduce the sensor sensitivity ( $\varepsilon$ in $\left.{ }^{\circ} \mathrm{C} . \mathrm{g} / \mathrm{W}\right)$ by considering the thermal heat capacity of the material sample and its mass [4-5, 10]. This type of transient calibration can be realized inside the reactor by withdrawing the sensor from the experimental channel.

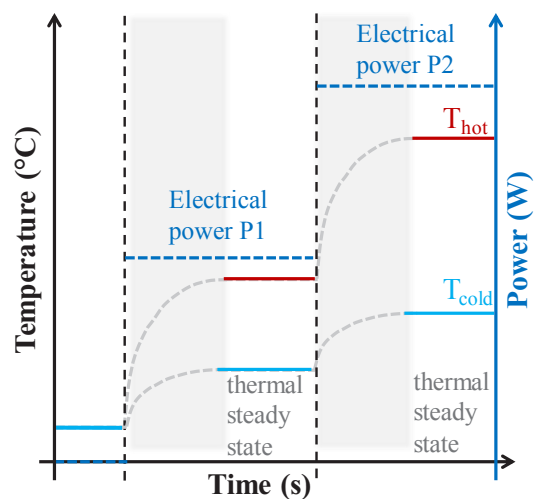

Fig. 4. Scheme of the calibration operating protocol of a calorimetric cell with a heater.

In the case of a calorimeter integrating heaters such as differential calorimeters, the calibration method is based on the analysis of successive steady thermal phases (cf. Fig. 4). In that case, different nuclear heating rates are experimentally simulated inside a localized zone of the sensor (in general inside the head center and more recently inside the rod and the base too [21]) by generating different consecutive values of a heat source by means of electrical current injections (increment by increment after reaching a steady thermal state) [2, 20-23]. Thanks to this operating protocol applied to each calorimetric cell, a curve representing the difference of the average steady temperatures versus the injected electrical power is plotted. This curve corresponds to the calibration curve of each calorimetric cell. The calorimetric cell sensitivity in ${ }^{\circ} \mathrm{C} / \mathrm{W}$ can be deduced. This calibration procedure is applied under non irradiation conditions in laboratory in general but it can be used inside reactors.

\section{IN-PILE MEASUREMENTS}

During the irradiation campaigns, the nuclear heating rate can be determined by measuring steady temperature difference(s) for the two categories of calorimeters. However, the differential calorimeters allow the application of three measurement methods due to its several calorimetric cells and its heaters whereas a single-cell calorimeter is associated to one method. In the case of a single-cell calorimeter without heater, the determination of the nuclear heating value at an axial position depends on the sensor sensitivity obtained during the calibration step and on the steady temperature difference measured at this position. The nuclear heating value is defined by the ratio between $\Delta \mathrm{T}$ and $\varepsilon[10]$.

In the case of differential calorimeters, three in-pile measurement methods can be applied [5, 16-17] (cf. Fig. 5). For the first one the heaters inside the cells are not used. The steady temperature difference of each cell is measured consecutively when the two cells are at the same axial position. For the two other methods, an electrical current is applied inside one heater (located inside the sample cell for the addition method or located inside the reference cell for the zero method). These two methods are more time consuming and have range limitations induced by electrical current limitations.

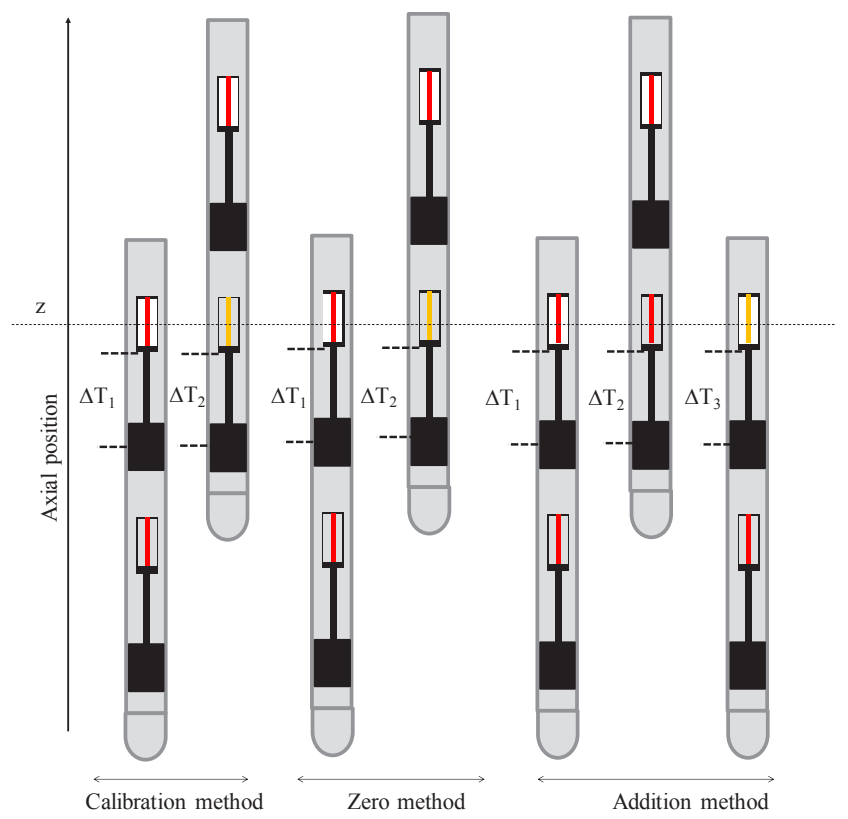

Fig. 5. Scheme of the three in-pile measurement methods for a differential calorimeter composed of two superposed calorimetric cells (passive heater in red, active heater in orange). 
For the zero method, an electrical current is applied inside the reference cell heater to obtain the same steady temperature difference for the two cells at the same axial position.

For the addition method, three steps are required. The first and the second steps are identical to the calibration method steps. During the third step, the sample cell is moved to its initial position and an electrical current is applied inside its heater in order to increase its steady temperature difference.

For the three methods, the nuclear heating value at a specific axial location is deduced by considering the calibration curve coefficients of the two cells, the steady temperature difference measurements of the two cells at this axial position during the different steps and a heat balance associated with the calorimetric cells (by taking into account of the nuclear heating and the additional energy induced by the heaters in the case of the zero and addition methods) [2-5].

\section{EXAMPLES OF PROBES INTEGRATING CALORIMETERS}

Current in-pile instrumentation generally consists of multisensor probes where calorimeters are associated with other sensors. Three probes are described in Figure 6.

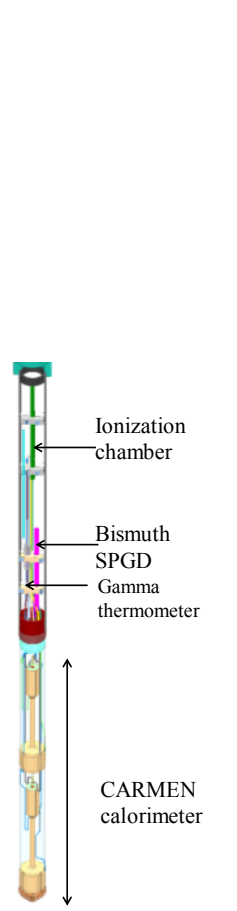

(a)

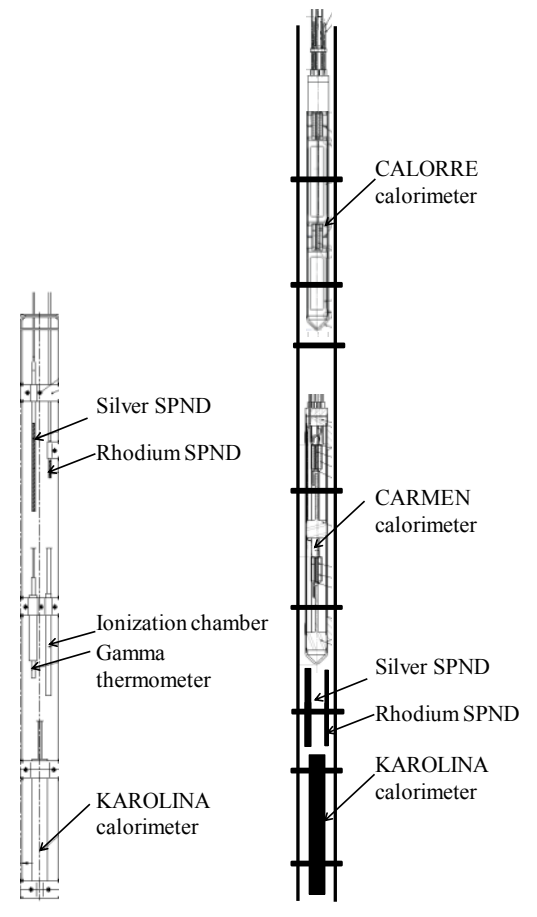

(c)
Fig. 6. Diagrams of the three multi-sensor probes: CARMEN 1P (a), GAMMA MAJOR probe (b) and INTERCAL (c).

The first probe (a), called CARMEN 1P and developed in the framework of the IN-CORE program, hosts different sensors dedicated to photon measurements and nuclear heating: a differential calorimeter, a gamma thermometer, an ionization chamber and a SPGD (bismuth) [2, 5]. The differential calorimeter used has the same global design as CALMOS type calorimeter but different size characteristics of the cell rod and base in order to have a more sensitive sensor for OSIRIS reactor periphery quantifications (up to $2 \mathrm{~W} / \mathrm{g}$ ). In fact, the probe called CALMOS, composed of a differential calorimeter, a SPND (rhodium) and a mechanical displacement system was designed to perform in-core measurement in OSIRIS (up to $13 \mathrm{~W} / \mathrm{g}$ ) [16-
18]. The main aim of the experiments realized with CARMEN 1P inside several experimental channels of OSIRIS periphery was to propose an advanced radiation measurement methodology by coupling this probe with a second probe called CARMEN $1 \mathrm{~N}$ focusing on neutron detections (probe dedicated to neutron measurements including two fission chambers $(242 \mathrm{Pu}$ and $235 \mathrm{U})$, an ionization chamber and a SPND (rhodium)).

The second probe (b) designed in the framework of the GAMMA MAJOR program focuses on neutron and photon measurements by means of KAROLINA calorimeter, a gamma thermometer, an ionization chamber and two SPNDs (silver and rhodium) [2-4]. The major aim of the experiments carried out inside different experimental channels of MARIA reactor was to test the Polish calorimeter and to use experimental results to validate new numerical works.

The last probe (c), called INTERCAL and developed in the framework of the IN-CORE program [22], contains two SPNDs (silver and rhodium) and three types of calorimeters: KAROLINA calorimeter, CARMEN type calorimeter (with a rod dimension change and thus new calibration coefficients) and CALORRE calorimeter. The main aim of the experiments carried out inside one experimental channel of MARIA reactor was to study the CALORRE calorimeter for the first time under real irradiation conditions, to validate it and to compare its response with the two conventional calorimeters.

\section{CONCLUSIONS}

Calorimetric measurements are crucial in fission reactors to quantify the nuclear heating rate. This relevant parameter is required to design loops, devices, irradiation experiments and conditions but it can participate to monitor other parameters too. Nuclear heating rate measurements can be realized by means of calorimeters such as single-cell calorimeters and differential calorimeters. According to their design and their assembly into a probe, these kinds of sensors can be used as a fixed technique (USA gamma thermometer, French five-stage calorimeter) or as a mobile technique (French CALMOS, CARMEN and INTERCAL probes for instance). The present tendency is the development of multi-sensor probes allowing the measurements of several parameters simultaneously and hosting calorimeters.

The choice of the calorimeter can be done by considering several criteria such as the nuclear heating rate level, the sensor size and sensitivity, the calibration operating protocol, the inpile measurement methods, and the sensor displacement, among other factors.

\section{REFERENCES}

[1] H. Amharrak et al., State of the art on nuclear heating in a mixed $(\mathrm{n} / \gamma)$ field in research reactors, Nuclear Instruments and Methods in Physics Research A, 2014, Volume 749, (2014) p. 57-67.

[2] J. Brun et al., Responses of Single-Cell and Differential Calorimeters: from Out-of-Pile Calibration to Irradiation Campaigns, IEEE TNS, Vol. 63, NO. 3, June 2016, p. 1630-1639.

[3] M. Tarchalski et al., Comparison Of Calculation Scheme With Multisensor Prompt And Delayed Nuclear Heating Measurements In The Maria Reactor, ENC 2016, 9-13 October 2016, Warsaw, Poland.

[4] M. Tarchalski, Nuclear heating measurements in the MARIA reactor and implementation of neutron and photon calculation scheme, thesis 2016. 
[5] D. Fourmentel et al., Nuclear Heating Measurements in Material Testing Reactor: a Comparison Between a Differential Calorimeter And a Gamma Thermometer, IEEE TNS, Vol. 60, Issue:1, Part:2, 2013, p. 328 - 335.

[6] B. Yu. Volkov, Qualification of Power Determination for F\&M Experiments in Research Reactors, RRFM 2016, 13-17 March 2016, Berlin, Deutschland.

[7] A.W. Boyd, International Intercomparison of Calorimeters, A report of measurements made in the reactor Melusine at CEN Grenoble, March 1970.

[8] M. Varvayanni et al., A point kernel model for the energy deposited on samples from gamma radiation in a research reactor core, Annals of Nuclear Energy 35 (2008) p. 2351-2356.

[9] Wang Qiu-kuan et al., Advanced Measuring (Instrumentation)Methods for Nuclear Installations: A Review, Science and Technology of Nuclear Installations, Volume 2012, Article ID 672876, 9 pages.

[10]Don W. Miller et al., A review on gamma thermometer applications in nuclear reactors, The 12th International Topical Meeting on Nuclear Reactor Thermal Hydraulics (NURETH-12), Pittsburgh, Pennsylvania, USA, September 30-October 4, 2007.

[11] GE-Hitachi Nuclear Americas, LPRM Calibration oand Power Shape Monitoring, report NEDO-33197-A, Revision 3, Class I, October 2010.

[12] R. Raghavan et al., Application of the gamma thermometer as BWR fixed in-core calibration system, Proceedings of NEA Specialists' Meeting on Incore Instrument and Core Assessment, Mito-shi, Japan, October 14-17 (1996).

[13] Y. Yamauchi et al., Development of improved core monitoring system, Journal of Power and Energy Systems, Vol. 2, No. 2, 2008, pp.712-719.

[14]J. Rempe et al., Status Report on Efforts to Enhance Instrumentation to Support Advanced Test Reactor Irradiations, INL Report, December 2012.

[15]J. P. Waring et al., Recent Reactor Testing and Experience with Gamma Thermometers, IEEE TNS, Vol. 30, Issue: 1, Feb. 1983, p. 791 - 794.

[16]H. Carcreff et al., Development, Calibration and Experimental Results Obtained with an Innovative Calorimeter (CALMOS) for Nuclear Heating Measurements, IEEE TNS, Vol. 59, №4, p. 1369-1376, 2012.

[17]H. Carcreff et al., "First In-Core Measurement Results Obtained with the Innovative Mobile Calorimeter CALMOS inside the OSIRIS Material Testing Reactor", IEEE TNS, Vol. 61, N4, pp. 1951-1962, 2014.

[18]H. Carcreff et al., First In-Core Simultaneous Measurements of Nuclear Heating and Thermal Neutron Flux Obtained With the Innovative Mobile Calorimeter CALMOS Inside the OSIRIS Reactor, IEEE Transactions on Nuclear Science, vol. 63, issue 5, pp. 2662-2670, 2016

[19]A. Péron et al., Simulation and Comparison of the Calorimeters Measuring the Nuclear Heating in the OSIRIS Reactor, with the TRIPOLI-4 MonteCarlo Code, IEEE Transactions on Nuclear Science, Volume: 62, Issue: 3 , June 2015, pp. $1218-1225$.

[20]J. Brun et al., Comparison of the thermal calibration response of two calorimetric cells dedicated to nuclear heating measurements IEEE TNS, Vol. 61, Issue: 4, Aug. 2014, p. 2254-2261, 2014.

[21] C. De Vita et al., Study of the Influence of Heat Sources on the Out-ofPile Calibration Curve of Calorimetric Cells Used for Nuclear Energy Deposition Quantification, IEEE TNS, Vol.63, Issue: 4, Aug. 2016, p. 2323 -2330 .

[22]J. Brun, et al., Irradiation campaign in MARIA reactor of an innovative calorimeter. Comparison of its nuclear heating measurements with two kinds of classical sensors. ANIMMA « Advancements for Nuclear Instrumentation and Measurement Methods and their Applications », 20 23 June 2017, Liège, Belgium.

[23] A. Volte et al., Study of the Flow Temperature and Ring Design Influence on the Response of a new Reduced-Size Calorimetric cell for Nuclear Heating Quantification. ANIMMA « Advancements for Nuclear Instrumentation and Measurement Methods and their Applications » 20 23 June 2017, Liège, Belgium. 